\title{
Takotsubo Cardiomyopathy Mimicking Myocardial Infarction in a Man with Myasthenic Crisis: A Case Report and Literature Review
}

\author{
Taylor M. Douglas ${ }^{1}$, Perry Wengrofsky ${ }^{1}$, Syed Haseeb ${ }^{1}$, Eric Kupferstein ${ }^{2}$, Pramod Theetha Kariyanna ${ }^{2}$, \\ Jacob Schwartz ${ }^{1}$, Louis Salciccioli ${ }^{2}$, Samy I. McFarlane ${ }^{1, "}$ \\ ${ }^{1}$ Department of Internal Medicine, State University of New York, Downstate Medical Center, Brooklyn, N.Y, U.S.A-11203 \\ ${ }^{2}$ Division of Cardiovascular Disease, Department of Internal Medicine, State University of New York, \\ Downstate Medical Center, Brooklyn, N.Y, U.S.A-11203 \\ *Corresponding author: Samy.mcfarlane@ downstate.edu
}

Received August 13, 2018; Revised October 02, 2018; Accepted October 11, 2018

\begin{abstract}
Takotsubo Cardiomyopathy is a syndrome characterized by transient and reversible regional myocardial dysfunction in the absence of obstructive coronary artery disease classically resulting in ventricular apical ballooning. It has a strong female predominance with onset generally in seventh decade of life, with hypothesized pathophysiology related to excess of catecholaminergic stimulation, particularly during episodes of physical or emotional stress. Takotsubo cardiomyopathy has been previously reported during myasthenic crisis, the acute deterioration of myasthenia gravis typically involving respiratory failure that is also associated with physical or emotional stress. We present the case of an atypically young male patient with classical takotsubo cardiomyopathy in the setting of myasthenic crisis after thymectomy initially concerning for ST segment elevation myocardial infarction, and a review of the literature of takotsubo cardiomyopathy in myasthenic crisis.
\end{abstract}

Keywords: myasthenic crisis, takotsubo cardiomyopathy, male, thymectomy

Cite This Article: Taylor M. Douglas, Perry Wengrofsky, Syed Haseeb, Eric Kupferstein, Pramod Theetha Kariyanna, Jacob Schwartz, Louis Salciccioli, and Samy I. McFarlane, "Takotsubo Cardiomyopathy Mimicking Myocardial Infarction in a Man with Myasthenic Crisis: A Case Report and Literature Review." American Journal of Medical Case Reports, vol. 6, no. 9 (2018): 184-188. doi: 10.12691/ajmcr-6-9-4.

\section{Introduction}

Takotsubo Cardiomyopathy (TC), also referred to as "broken-heart" syndrome and "stress cardiomyopathy", is a syndrome characterized by transient and reversible regional myocardial dysfunction with various patterns of regional hyperkinesis and hypokinesis in the absence of underlying obstructive coronary artery disease (CAD), most classically with left ventricular (LV) apical hypokinesis and basal hyperkinesis resulting in apical ballooning $[1,2]$. TC has been shown to have an overwhelming female predominance and average age of onset of 66 , with strong temporal associations to emotional and physical stress, with postulated mechanisms centered around catecholamine-induced myocardial injury and catecholamine-mediated vascular spasm and dysfunction causing myocardial stunning $[3,4]$. TC is among the most commonly reported cardiac manifestations of Myasthenia Gravis (MG), a neuromuscular autoimmune disease with auto-antibodies to nicotinic acetylcholine receptors (anti-AChR) primarily affecting younger women and older men [5]. TC associated with MG most prominently appears during myasthenic crisis (MC), an acute deterioration of MG typically brought on by a physical or emotional stressor that usually involves respiratory muscles resulting in respiratory failure and the need for mechanical ventilation [6]. Previous reports of $\mathrm{MC}$ triggered TC demonstrate a female predominance with variable antecedent history of thymectomy [6]. We present the first reported case of TC in MC in a male patient after thymectomy.

\section{Case Report}

A 49-year-old male with a past medical history significant for MG with thymectomy 5 months prior to presentation was transferred to our institution after presenting to an outside hospital with impending respiratory failure concerning for acute MC. At the outside hospital, the patient was intubated, and started on pyridostigmine, prednisone, and intravenous immunoglobulin (IVIg). Compared to baseline EKG (Figure 1), on day 3 of his hospitalization, electrocardiogram (EKG) revealed new $\mathrm{T}$ wave inversions V2-V6 and inferior limb leads II, III, aVF (Figure 2). 


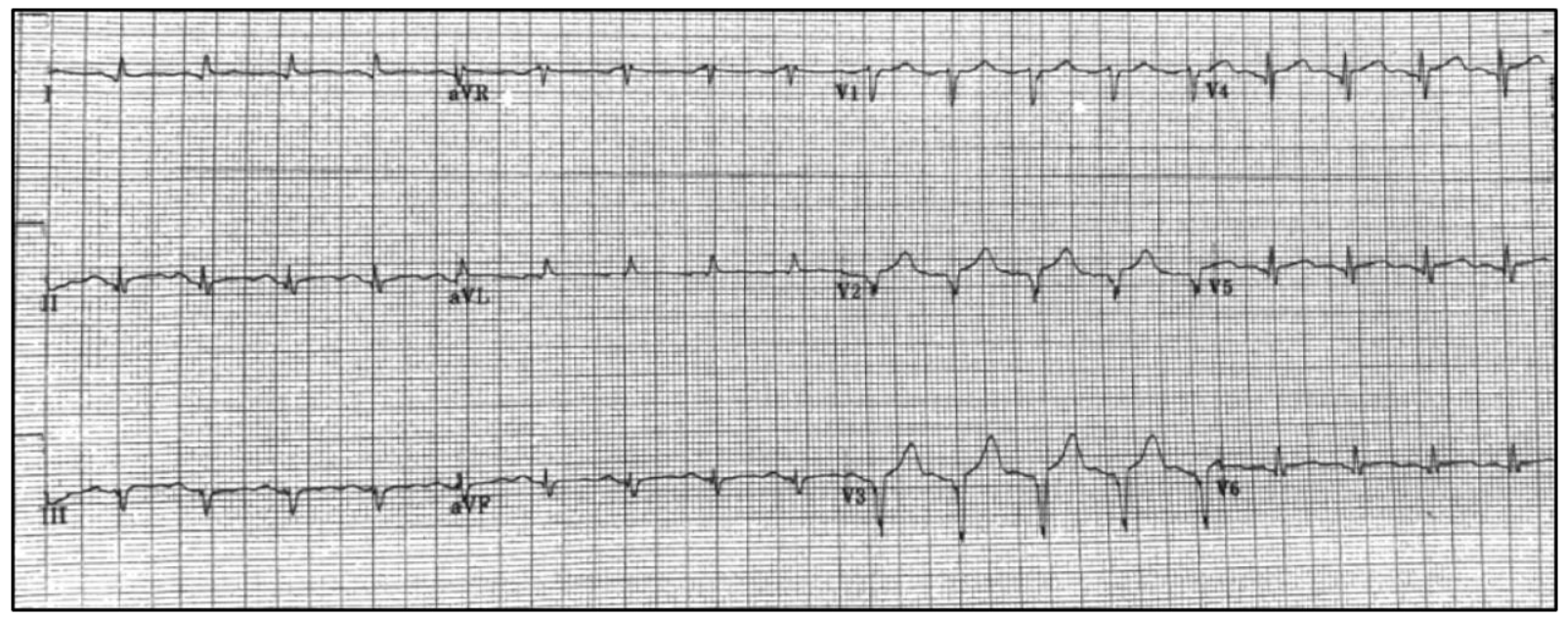

Figure 1. EKG at initial presentation. No anterolateral T-wave abnormality noted. The EKG reveals normal sinus rhythm, Q-waves in V1-V3 and T wave flattening in I and aVL

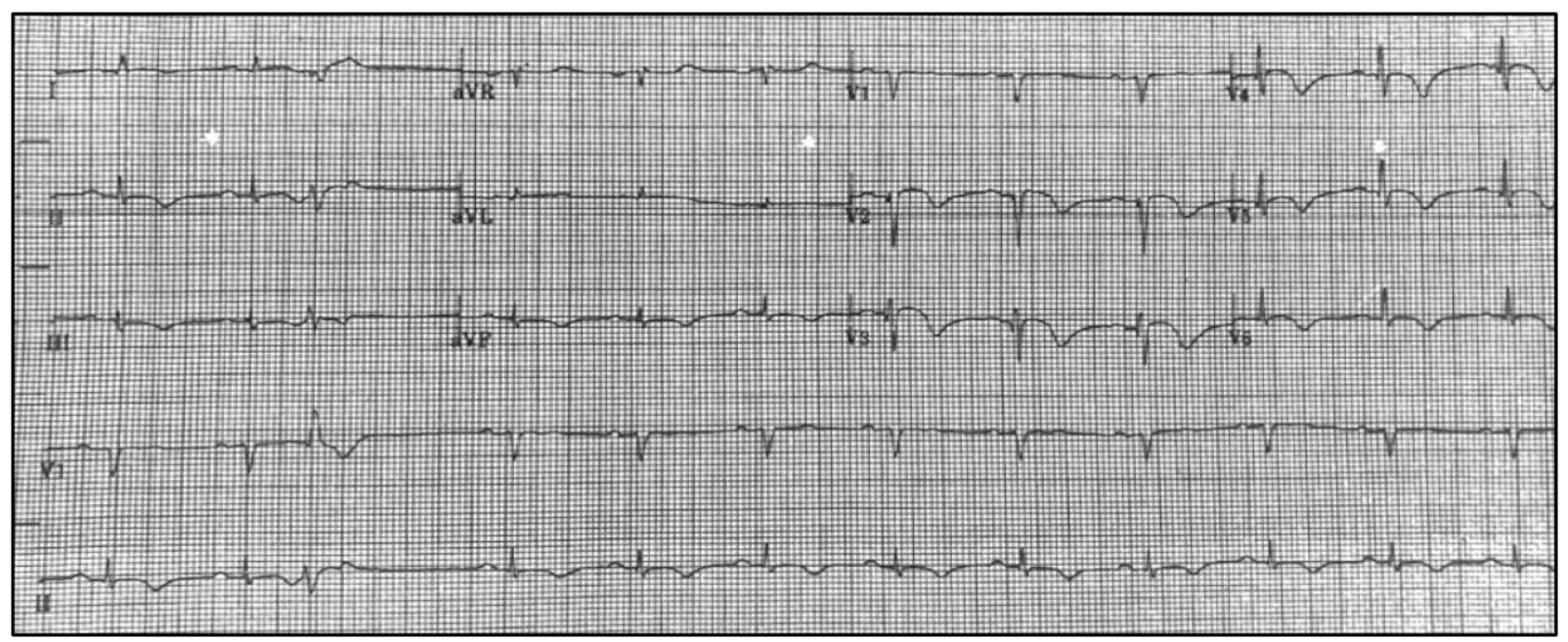

Figure 2. Subsequently obtained EKG revealing T wave inversions in precordial leads V2-V6 and inferior limb leads II, III, aVF

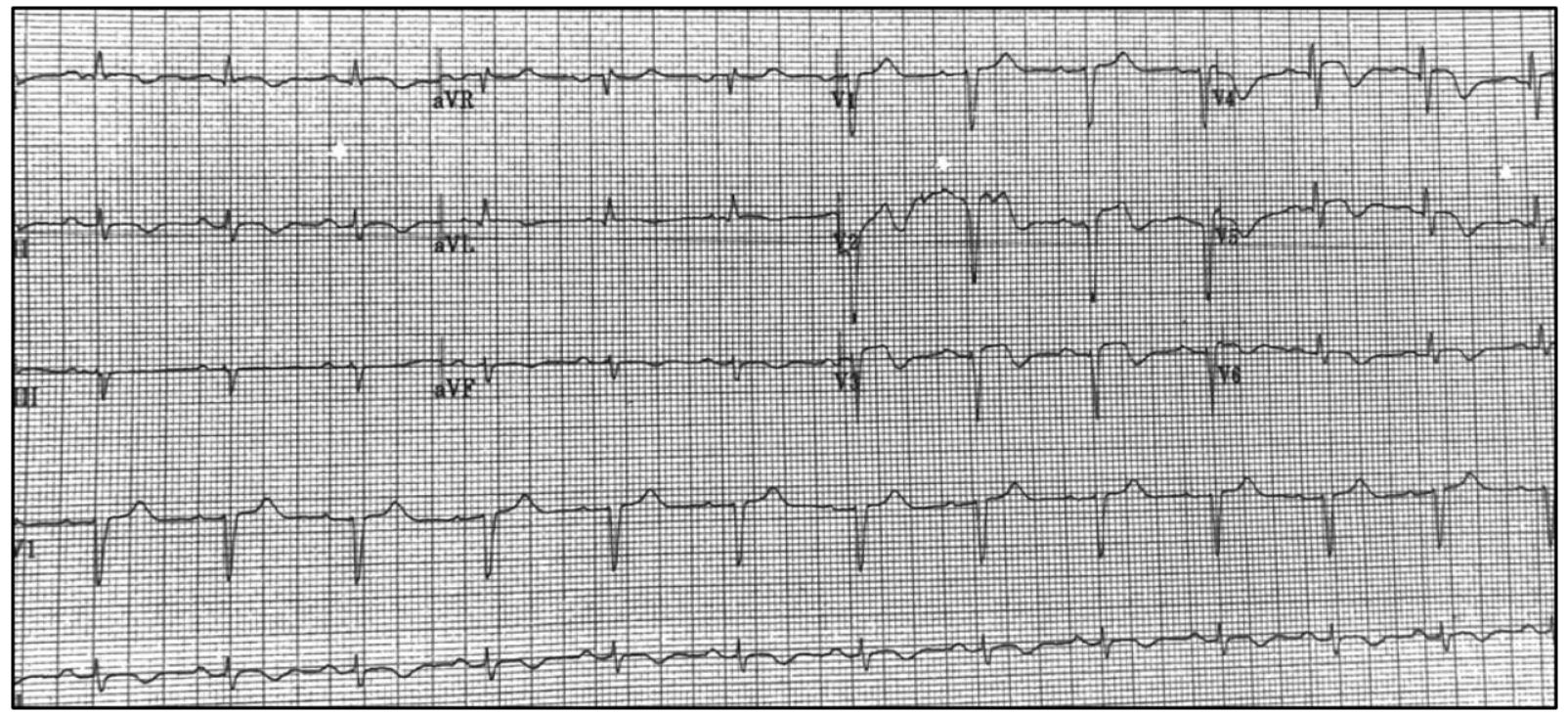

Figure 3. EKG revealing ST elevation in precordial leads (V2-V6).

Given concern for NSTEMI, intravenous heparin was started. The patient subsequently became hypotensive and was started on norepinephrine and then transitioned to phenylephrine due to tachycardia. Serial EKGs revealed mrore pronounced ST elevation in V2-V6 concerning for injury with persistent $\mathrm{T}$ waves inversions in inferior and precordial leads (Figure 3). Troponin levels uptrended, peaking at $2.47(\mathrm{ng} / \mathrm{mL})$. The Patient was transferred to our institution for emergent cardiac catheterization for suspected STEMI. 


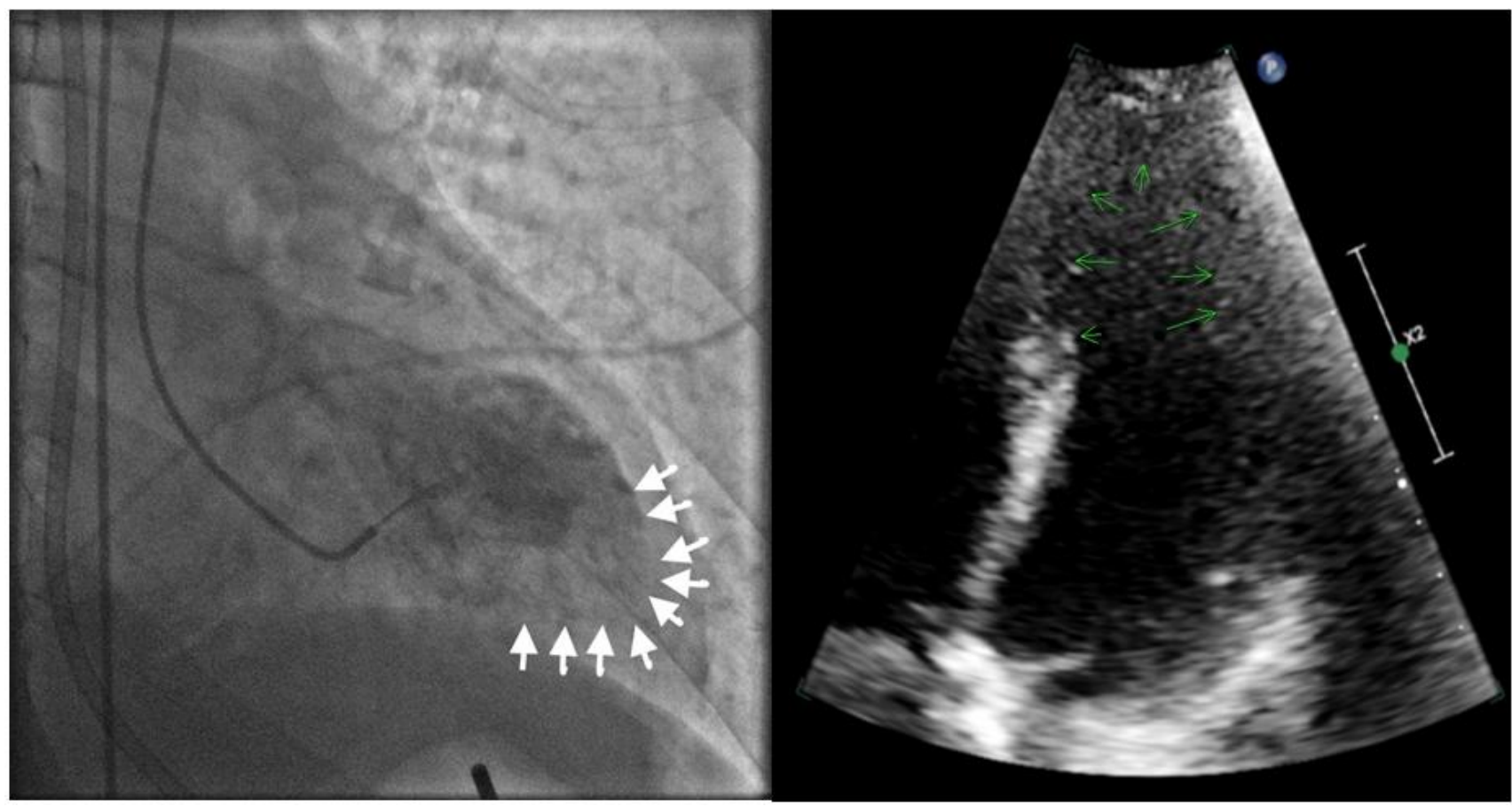

Figure 4. a (left): Ventriculogram revealing apical ballooning, b (right): TTE revealing hypokinesis of mid-distal anteroseptal, mid-apical, inferior, apex and lateral walls with apical ballooning.

Upon arrival, coronary angioprahy revealed widely patent coronaries with mild lumen abnormalities, an EF of 30 to $35 \%$ with severe hypokinesis and ballooning of the distal anterolateral/apical/inferior walls, suggestive of TC (Figure 4a). Transthoracic echocardiography (TTE) revealed severe hypokinesis of the apex along with the mid-distal anteroseptal, mid-apical, lateral and inferor walls with adequate contraction appreciated in the basal portions of the LV, consistent with TC (Figure 4b). Phenylephrine was titrated off and the patient remained stable without pressor support. IVIg, pyridostigmine, and prednisone were continued. Mechanical ventilation was continued with frequent suctioning for copious secretions perhaps exacerbated by pyridostigmine with monitoring of negative inspiratory force and forced vital capacity. The patient remained hemodynamically stable without overt signs of symptoms of acute decompensated heart failure, and was transferred back to the referring hospital for continued care. Decompensated heart failure did develop later however, was treated, and the patient was successfully extubated.

\section{Discussion}

TC in the setting of MC has been attributed to adverse catecholaminergic associated with the severe stress precipitated by $\mathrm{MC}$, and to the temporal relationship that has been extensively documented between physical or emotional stress, the onset of TC and the reversibility of $\mathrm{TC}$ with resolution of antecedent stressor.

While no specific pathophysiological mechanism of TC has been elucidated, evidence points to the role of excessive sympathetic and catecholaminergic stimulation given the robust temporal association with emotional or physical precipitant events, association with conditions of catecholaminergic excess like pheochromocytoma, and the ballooning patterns seen on cardiac imaging after intravenous infusion of catecholamines and beta-agonists [7]. Four different morphologies of TC have been described; the classical type featuring apical ballooning with basal hyperkinesis; the mid-ventricular type featuring basal hyperkinesis with mid-ventricular hypokinesis and normal to hyperkinetic apex; the basal or inverted type featuring basal and mid-ventricular hypokinesis with apical hyperkinesis; the focal type featuring hypokinesis of a focal myocardial segment [8].

The distribution of hypo- and hyper-kinetic myocardium in the setting of increased adrenergic stimulation raises the question of potential regional catecholamine-mediated effects on myocardium and coronary circulation. Beta adrenergic receptors (BAR), the G-protein coupled adrenergic receptors of the sympathetic nervous system, and sympathetic nerve endings are unequally distributed in human hearts, with a prominent apical-basal gradient containing a decreasing density of sympathetic nerve endings in the apical myocardium as compared to basilar myocardium, and increasing density of BAR in the apical myocardium as compared to basilar myocardium $[9,10]$. The discrepant ratio of sympathetic nerve ending densities, and thus norepinephrine, and beta receptors in the basal and apical myocardium results in increased activity of circulating epinephrine on BAR in the apical myocardium [11]. High concentrations of epinephrine have been shown to induce $32 \mathrm{AR}$ to switch its G-protein coupling from stimulatory G-protein (Gs) to inhibitory G-protein (Gi), known as ligand-directed trafficking or biased agonism [12]. Paur et al. demonstrated in a rat model that high dose epinephrine infusion mimicking the catecholamine response to acute stress generated the classical reversible apical ballooning pattern of TC from apical and mid-ventricular hypokinesis with preserved to enhanced basal contractility, but such myocardial contractility patterns were not reproduced with equivalent high dose 
norepinephrine acute stress bolus [13]. Furthermore, it was demonstrated that there is basal-apical gradient of catecholamine responsiveness to BAR subtype, with apical cardiomyocytes having a higher density of B2AR and greater B2AR-induced sensitivity compared with basal cardiomyocytes [13].

Confounding the catecholaminergic-mediated myocardial dysfunction of TC are the catecholamine-mediated changes in coronary vascular tone and the parasympathetic dysfunction seen in MC. The autonomic changes of MC are characterized by parasympathetic dysfunction, with lowered parasympathetic excitatory postsynaptic potentials suspected to be secondary to damage and blockade from the nicotinic anti-AChR on postsynaptic ganglion AChR [14,15]. Elevated circulating levels of catecholamines from the physical stress of MC compounded with blunted parasympathetic innervation enables dramatic sympathetic and adrenergic tone on the coronary vasculature, both on the epicardial vessels and microcirculatory system. Reversible coronary epicardial and microcirculatory dysfunction has been confirmed in TC with transient improvements in regional myocardial dysfunction, wall motion score index, wall motion defect length, LV end systolic volume (LVESV) and EF with coronary vasodilation from infusion of $\mathrm{ACh}$ or adenosine $[16,17]$.
To the best of our knowledge, this is the first case of $\mathrm{TC}$ in $\mathrm{MC}$ in a male patient after thymectomy. TC in MC has been previously reported [18-35], however there are only three previous reports of male patients with $\mathrm{TC}$ in MC [19,23,32], and there are only two previous reports of TC in MC after thymectomy, with both patients being female [21,30]. Review of the literature demonstrats a strong female predominance (16:3), with an average age of onset of 64 years of age, considerably older than our patient. Demographic and LV function data from previous case reports are summarized below in Table 1. EKG changes seen in previous cases of TC during MC demonstrate a varying pattern of ST segment elevations and/or $\mathrm{T}$ wave inversions, most prominently in the precordial leads, most specifically the anterolateral leads. In the case of our patient, ST segment elevation followed $\mathrm{T}$ wave inversion. Previous analysis of EKG evolution in patients with TC show distinct patterns of evolution, with $\mathrm{T}$ wave inversions generally following resolution of antecedent ST elevation, and initial $\mathrm{T}$ wave inversion accompanied by QT prolongation without concurrent ST elevation [36]. Our case displayed initial EKG changes of $\mathrm{T}$ wave inversions, followed by ST elevation, and persistence of $\mathrm{T}$ wave inversions after resolution of ST elevation.

Table 1. Previously published cases of Takotsubo cardiomyopathy in patients with myasthenia crisis

\begin{tabular}{|c|c|c|c|c|}
\hline Year, Case & Age (years) & Gender & Thymoma/ Thymectomy & TTE \\
\hline 2004, Arai et al. [18] & 83 & F & None & Extensive akinesis - apex \\
\hline 2005, Sousa et al. [19] & 64 & M & None & $\begin{array}{l}\text { Dyskinesis - apex } \\
\text { Akinesis - middle segments of all wall } \\
\text { Hypokinesis - anterior and septal basal wall segments }\end{array}$ \\
\hline 2008, Hirose et al. [20] & 63 & $\mathrm{~F}$ & None & LV wall motion asynergy \\
\hline 2009, Sasidharan et al. [21] & 40 & $\mathrm{~F}$ & Thymectomy & $\begin{array}{l}\text { Dilated LV, severe LV dysfunction } \\
\text { Akinesis - apical segments } \\
\text { Hyperkinesis - basal segments }\end{array}$ \\
\hline 2010, Beydoun et al. [22] & 60 & $\mathrm{~F}$ & None & $\begin{array}{l}\text { LVEF 32\% } \\
\text { Ballooning - apex } \\
\text { Hyperdynamic - proximal apex* }\end{array}$ \\
\hline 2011, Bansal et al. [23] & 77 & M & None & $\begin{array}{l}\text { Severe \& diffusely decreased LV function, regional } \\
\text { variations } \\
\text { Hypokinesis - distal > proximal segments }\end{array}$ \\
\hline 2011, Gautiera et al. [24] & 82 & $\mathrm{~F}$ & - & $\begin{array}{l}\text { Dyskinesis - antero-medium \& late-medium-high } \\
\text { walls } \\
\text { Enhancement - late postero-basal enhancement } \\
\text { EF of } 56 \% \text { with conserved global kinetics** }\end{array}$ \\
\hline 2012, Wong et al. [25] & 64 & $\mathrm{~F}$ & - & $\begin{array}{l}\text { Impaired LV with EF of } 30 \% \\
\text { Ballooning - apex } \\
\text { Hyperkinesis - basal region* }\end{array}$ \\
\hline 2012, Nishinarita et al. [26] & 52 & $\mathrm{~F}$ & Thymoma & $\begin{array}{l}\text { Akinesis - apex } \\
\text { Hyperkinesis - proximal }\end{array}$ \\
\hline 2012, Mayor-Gomez et al. [27] & 83 & $\mathrm{~F}$ & - & Dyskinesis - apex \\
\hline 2013, Valbusa et al. [28] & 75 & $\mathrm{~F}$ & None & $\begin{array}{l}\text { Ballooning - apex } \\
\text { Impaired LV function } \\
\text { Mild MR and AR }\end{array}$ \\
\hline 2013, Anand et al. [29] & 50 & $\mathrm{~F}$ & None & Hypokinesis - anterior \& distal septum, apex \\
\hline 2014, Thanaviratananich et al. [30] & 42 & $\mathrm{~F}$ & Thymectomy & $\begin{array}{l}\text { Severe hypokinesis - global w/relative sparing of } \\
\text { apex LVEF of } 15 \%\end{array}$ \\
\hline 2014, John et al. [31] & 34 & $\mathrm{~F}$ & None & $\begin{array}{l}\text { Hyperkinesis - apex } \\
\text { Akinesis - basal wall }\end{array}$ \\
\hline 2015, Harries et al. [32] & 70 & M & None & $\begin{array}{l}\text { Hypokinesis - apex } \\
\text { Preserved - basal segment }\end{array}$ \\
\hline 2016, Cuevas et al. [33] & 69 & $\mathrm{~F}$ & None & $\begin{array}{l}\text { Hypokinesis - antero-/infero-apical } \\
\text { Hyperkinesis - basal segment* }\end{array}$ \\
\hline 2017, Battineni et al. [34] & 69 & $\mathrm{~F}$ & None & $\begin{array}{l}\text { Mid-ventricular variant TC } \\
\text { Reduced EF of } 25 \%\end{array}$ \\
\hline 2018, Jolly et al. [35] & 75 & $\mathrm{~F}$ & None & $\begin{array}{l}\text { Hypokinesis - apex and septal segments } \\
\text { LVEF of } 35 \%\end{array}$ \\
\hline
\end{tabular}

Key: *Ventriculogram findings. **Cardiac MRI findings. $\mathrm{F}=$ Female. $\mathrm{M}=$ Male. 
In summary, we present the case of a male patient with $\mathrm{TC}$ in the setting of $\mathrm{MC}$ after thymectomy. TC remains a rare complication of $\mathrm{MG}$ and $\mathrm{MC}$ and is uncommon in male patients and those with a history of thymectomy. Considering the prominent role of catecholamines and the asymmetric adrenergic stimulation underlying the pathophysiology of TC, understanding the overlap between MG anti-AChR and the blunted parasympathetic stimulation present in MG could serve as a potential area of investigation into the mechanism of $\mathrm{TC}$ in $\mathrm{MC}$.

\section{Acknowledgements}

This work is supported, in part, by the efforts of Dr. Moro O. Salifu M.D., M.P.H., M.B.A., M.A.C.P., Professor and Chairman of Medicine through NIH Grant number S21MD01247.

\section{References}

[1] Bybee KA, Kara T, Prasad A, Lerman A, Barsness GW, Wright RS, Rihal CS. Systematic review: transient left ventricular apical ballooning: a syndrome that mimics ST-segment elevation myocardial infarction. Annals of internal medicine. 2004 Dec 7 141(11): 858-65.

[2] Satoh H. Takotsubo-type cardiomyopathy due to multivessel spasm. Clinical aspect of myocardial injury: from ischemia to heart failure. 1990

[3] Templin C, Ghadri JR, Diekmann J, Napp LC, Bataiosu DR, Jaguszewski M, Cammann VL, Sarcon A, Geyer V, Neumann CA, Seifert B. Clinical features and outcomes of Takotsubo (stress) cardiomyopathy. New England Journal of Medicine. 2015 Sep 3 373(10): 929-38

[4] Nef HM, Möllmann H, Kostin S, Troidl C, Voss S, Weber M, Dill T, Rolf A, Brandt R, Hamm CW, Elsässer A. Tako-Tsubo cardiomyopathy: intraindividual structural analysis in the acute phase and after functional recovery. European heart journal. 2007 Mar 29; 28(20): 2456-64.

[5] Shivamurthy P, Parker M. Cardiac manifestations of myasthenia gravis: A systematic review. IJC Metabolic and Endocrine. 2014 5: 3-6.

[6] Finsterer J, Stollberger C. Stress from myasthenic crisis triggers Takotsubo (broken heart) syndrome. International Journal of Cardiology. 2016; 203: 616-617.

[7] Ghadri J, Wittstein IS, Prasad A, et al. International expert consensus document on Takotsubo Syndrome (Part I): Clinical characteristics, diagnostic criteria, and pathophysiology. European Heart Journal. 2018; 39: 2032-2046

[8] Templin C, Ghadri JR, Diekmann J, et al. Clinical Features and outcomes of takotsubo (stress) cardiomyopathy. New England Journal of Medicine. 2015; 373: 929-938.

[9] Lyon AR, Rees PS, Prasad S, et al. Stress (Takotsubo) cardiomyopathy - a novel pathophysiological hypothesis to explain catecholamine-induced acute myocardial stunning. Clin Pract Cardiovasc Med. 2008; 5: 22-29.

[10] Kawano H, Okada R, Yano K. Histological study on the distribution of autonomic nerves in the human heart. Heart Vessels. 2003; 18 : 32-39.

[11] Gopalakrishnan P, Zaidi R, Sardar MR. Takotsubo cardiomyopathy: Pathophysiology and role of cardiac biomarkers in differential diagnosis. World Journal of Cardiology. 2017; 9(9): 723-730.

[12] Heubach JF, Ravens U, Kaumann AJ. Epinephrine activates both Gs and Gi pathways, but norepinephrine activates only the Gs pathway through human beta2-adrenoceptors overexpressed in mouse heart. Mol Pharmacol. 2004; 65: 1313-1322.

[13] Paur H, Wright PT, Sikkel MS, et al. High levels of circulating epinephrine trigger apical cardiodepression in a B2adrenoceptor/Gi-dependent manner: A new model of Takotsubo cardiomyopathy. Circulation. 2012; 126(6): 697-706.
[14] Peric S, Rakocevic-Stojanovic V, Nisic T, et al. Cardiac autonomic control in patients with myasthenia gravis and thymoma. Journal of the Neurological Sciences. 2011; 307: 30-33.

[15] Benjamin RN, Aaron S, Sivadasan A, et al. The spectrum of autonomic dysfunction in myasthenic crisis. Annals of Indian Academy of Neurology. 2018; 21(1): 42-48.

[16] Verna E, Provasoli S, Ghiringhelli S, et al. Abnormal coronary vasoreactivity in transient left ventricular apical ballooning (takotsubo) syndrome. International Journal of Cardiology. 2017; 250: 4-10.

[17] Galiuto L, De Caterina AR, Porfidia A, et al. Reversible coronary microvascular dysfunction: a common pathogenetic mechanism in Apical Ballooning or Tako-Tsubo Syndrome. European Heart Journal. 2010; 31: 1319-1327.

[18] Arai M, Ukigai H, Miyata H. A case of transient left ventricular ballooning ("Takotsubo"-shaped cardiomyopathy) developed during plasmapheresis for treatment of myasthenic crisis. Rinsho shinkeigaku= Clinical neurology. 2004 Mar; 44(3): 207-10.

[19] Sousa JM, Knobel M, Buchelle G, et al. Transient ventricular dysfunction (Takotsubo cardiomyopathy). Arquivos brasileiros de cardiologia. 2005 Apr; 84(4): 340-2.

[20] Hirose K, Yamaguchi H, Oshima Y, et al. Severe respiratory failure and torsades de pointes induced by disopyramide in a patient with myasthenia gravis. Internal Medicine. 2008; 47(19): 1703-8.

[21] Bijulal S, Harikrishnan S, Namboodiri N, e al. Unusual association of diseases/symptoms: Tako-tsubo cardiomyopathy in a patien with myasthenia gravis crisis: a rare clinical association. BMJ case reports. 2009; 2009

[22] Beydoun SR, Wang J, Levine RL, et al. Emotional stress as a trigger of myasthenic crisis and concomitant takotsubo cardiomyopathy: a case report. Journal of medical case reports. 2010 Dec; 4(1): 393

[23] Bansal V, Kansal MM, Rowin J. Broken heart syndrome in myasthenia gravis. Muscle \& nerve. 2011 Dec; 44(6): 990-3.

[24] Gautier P, Ravan R, Najjar M, et al. Tako-Tsubo syndrome during normal human immunoglobolin perfusion. InAnnales de Cardiologie et d'Angéiologie 2011 Nov (Vol. 60, No. 5, pp. 290-295).

[25] Wong CP, Chia PL. Recurrent takotsubo cardiomyopathy precipitated by myasthenic crisis. International journal of cardiology. 2012 Feb 23; 155(1): e11-2.

[26] Nishinarita R, Kawamura Y, Yasuda T, et al. A case of takotsubo cardiomyopathy leading to the diagnosis of myasthenia gravis. Journal of Cardiology Cases. 2012 Nov 1; 6(5): e141-4.

[27] Mayor-Gomez S, Lacruz F, et al. Myasthenic crisis and Takotsubo syndrome: a non-chance relationship. Revista de neurologia. 2012 Dec; 55(12): 725-8

[28] Valbusa A, Ingrassia S, Rosa GM, et al. Takotsubo cardiomyopathy and torsade de pointes in myasthenic crisis: be aware of QT prolongation. The American journal of emergency medicine. 2013 Dec 1; 31(12): 1717-8

[29] Anand US, Viswanathan S, Arulneyam J. Pulmonary edema in myasthenic crisis. Case reports in critical care. 2013; 2013.

[30] Thanaviratananich S, Katirji B, Alshekhlee A. Broken heart syndrome during myasthenic crisis. Journal of clinical neuromuscular disease. 2014 Mar 1; 15(3): 90-5.

[31] John A, Singh S, Singh A, et al. Reverse Takotsubo Cardiomyopathy from Myasthenic Crisis: A Case Report. Journal of Cardiovascular Diseases \& Diagnosis. 2014 Sep 4.

[32] Harries IB, Levoir H, Bucciarelli-Ducci C, et al. Case Report: Takotsubo cardiomyopathy in myasthaenia gravis crisis confirmed by cardiac MRI. BMJ case reports. 2015; 2015.

[33] Escutia Cuevas HH, Domínguez López R, et al.. Síndrome de takotsubo asociado a crisis miasténica. Presentación de un caso. Revista mexicana de cardiología. 2016 Oct 15; 27(3): 123-9.

[34] Battineni A, Mullaguri N, Thanki S, et al. A Case Report of Recurrent Takotsubo Cardiomyopathy in a Patient during Myasthenia Crisis. Case reports in critical care. 2017; 2017.

[35] Jolly G, Haidry A, Koulova A, et al. Myasthenia gravis crisis associated stress (takotsubo) cardiomyopathy. Journal of the American College of Cardiology. 2018 Mar 10; 71 (11 Supplement): A2355.

[36] Namgung J. Electrocardiographic Findings in Takotsubo Cardiomyopathy: ECG Evolution and Its Difference from the ECF of Acute Coronary Syndrome. Clinical Medicine Insights: Cardiology. 2014; 8: 29-34. 